\title{
Evaluación de salud reproductiva en perros machos mestizos enteros
}

\author{
Sánchez, A.E.; Pfeffer, M.E. \\ Núcleo de Investigaciones Aplicadas, Facultad de Medicina Veterinaria, \\ Universidad de Las Américas, Viña del Mar, Chile. \\ E-mail: asanchez@udla.cl
}

\begin{abstract}
Resumen
Sánchez, A.E.; Pfeffer, M.E.: Evaluación de salud reproductiva en perros machos mestizos enteros. Rev. Vet. 31: 2, 206-209, 2020. La salud reproductiva es un componente importante del bienestar animal. Se postula que los perros mestizos enteros de 5 o más años, independientemente de su peso vivo, presentarían más alteraciones subclínicas respecto de animales más jóvenes. El propósito de este estudio fue evaluar y caracterizar algunos parámetros de salud reproductiva en perros mestizos enteros de diferentes pesos vivos y edades, efectuando exámenes físicos y reproductivos, así como ultrasonografía de testículos y próstata. Se dispuso de 90 perros mestizos mayores de un año de edad, conformando seis grupos con tres categorías de peso vivo $(<15 \mathrm{~kg}, 15-25 \mathrm{~kg} \mathrm{y}>25 \mathrm{~kg})$ y dos categorías de edad ( $>1 / \leq 5$ años y $>5$ años). La evaluación se realizó a través de exámenes físico, reproductivo y ultrasonográfico, registrándose mayores frecuencias de anomalías testiculares (asimetría y flacidez) y mayor frecuencia de alteraciones de parénquima, especialmente heterogeneidad y presencia de focos anecoicos en perros $>5$ años, independientemente del peso vivo $(\mathrm{p}<0,05)$. En la evaluación prostática se registró mayor proporción de anomalías de ecotextura, asimetría lobular, contorno glandular irregular y presencia de focos anecoicos en los grupos de perros $>5$ años $(p<0,05)$. Cabe destacar que la prostatomegalia se observó en el $88 \%$ de los ejemplares $>5$ años y sólo en el $20 \%$ de los animales más jóvenes, independiente del peso vivo ( $\mathbf{p}<0,05)$, alcanzando un $54,4 \%$ en el total de la muestra.
\end{abstract}

Palabras clave: canino, próstata, testículo, salud, exámenes físicos, reproductivos y ultrasonográficos.

\begin{abstract}
Sánchez, A.E.; Pfeffer, M.E.: Evaluation of reproductive health in whole mestizo dogs. Rev. Vet. 31: 2, 206-209, 2020. Proposing that reproductive health is an important component of animal welfare and postulating the hypothesis that the reproductive health of whole mongrel dogs of 5 years or older, independent of their live weight, would present more subclinical alterations, with respect to younger animals. The purpose of this study was to evaluate and characterize some reproductive health parameters in whole mongrel dogs of different live weights and ages, through a reproductive physical examination and ultrasonographic examinations of testicles and prostate. By means of a sampling for convenience and with the consent of their owners, 90 mongrel dogs over 1 year of age were examined, forming six groups with three liveweight categories $(<15 \mathrm{~kg}, 15-25 \mathrm{~kg}$ and $>25 \mathrm{~kg})$ and two age categories $(>1 / \leq 5$ and $>5$ years). The evaluation was carried out by means of physical reproductive examination and ultrasonographic examination, recording higher frequencies of testicular anomalies (asymmetry and flaccidity) and higher frequencies of parenchymal alterations, especially heterogeneity and presence of anechoic foci in dogs $>5$ years, independent of live weight $(\mathrm{p}<0.05)$. In the prostatic evaluation, a higher proportion of ecotextural anomalies, lobular asymmetry, irregular glandular contour and presence of anechoic foci were recorded in groups of dogs $>5$ years $(\mathrm{p}<0.05)$. It should be noted that prostatomegaly was observed in $88 \%$ of the specimens $>5$ years and only in $20 \%$ of the younger animals, independent of live weight $(\mathrm{p}<0.05$ ), reaching $54.4 \%$ in the total sample.
\end{abstract}

Key words: dogs, prostate, testicle, health, physical, reproductive and ultrasound examinations.

\section{INTRODUCCIÓN}

En la sociedad actual, el trato que los seres humanos prodigan a los animales es una preocupación moral.

Recibido: febrero 2020 / Aceptado: mayo 2020
La ciencia del bienestar animal evalúa los comportamientos, las emociones y la salud ${ }^{5}$.

En caninos, resulta innovador proponer y socializar el concepto de salud reproductiva, particularmente en una dimensión preventiva, en orden de complementar lo declarado en cuanto a bienestar animal y tenencia 
responsable (Invest Cienc Anim 2: 33-36), considerando que un área de desarrollo para maximizar el bienestar de los perros sería la salud, mediante prevención ${ }^{21}$.

A lo anterior cabe agregar que los perros mestizos en la actualidad son un tipo de mascotas muy aceptadas ${ }^{7} \mathrm{y}$ que, al no tener un propósito reproductivo en su tenencia, la evaluación regular de la salud reproductiva en machos enteros no se considera prioritaria.

En los perros se describen distintos procesos patológicos que se asocian con la presencia y funcionalidad de los testículos. Ellos tienen por función la producción de espermatozoides y de hormonas reproductivas, cuyo fin es mantener la condición fisiológica del individuo en función de su potencial generación de progenie. Sin embargo, trastornos metabólicos, degenerativos, neoplásicos o infecciosos pueden afectar a las gónadas generando alteraciones de la salud ${ }^{15}$.

En perros la presencia de neoplasias testiculares es común, describiéndose prevalencias cercanas al $30 \%$ en pacientes geriátricos ${ }^{6}$. Además, el macho canino se caracteriza por poseer próstata como única glándula sexual accesoria, la cual fisiológicamente es dependiente de la testosterona testicular ${ }^{4,18}$.

Las enfermedades de la próstata son comunes en perros mayores de 6 años de edad, no castrados, destacándose que un alto porcentaje de estas patologías poseen características subclínicas, las que comúnmente son reportadas como hallazgos en la exploración ultrasonográfica ${ }^{1,4,20}$.

La evaluación de los órganos reproductivos del perro, se realiza mediante examen clínico y se complementa con ultrasonido, considerando que la ultrasonografía permite la evaluación precisa de las características del parénquima y de la arquitectura interna de dichas estructuras.

Postulamos la hipótesis de que la salud reproductiva de perros mestizos enteros de 5 o más años, independientemente de su peso vivo, presentaría mayores alteraciones subclínicas, respecto de animales más jóvenes.

El objetivo del presente estudio fue evaluar y caracterizar algunos parámetros de salud reproductiva en perros mestizos de diferentes pesos vivos y edades, a través de un examen físico reproductivo y exámenes ultrasonográficos de testículos y próstata.

\section{MATERIAL Y MÉTODOS}

Mediante un muestreo por conveniencia y con el consentimiento de sus propietarios, se examinaron 90 perros mestizos, clínicamente sanos, mayores de 1 año de edad.

El criterio de inclusión consideró la presencia de ambos testículos en el escroto, sin aumentos de volumen, así como la ausencia de signos evidentes de alteración prostática (hematuria, disuria, constipación o tenesmo).

El estudio se realizó en dependencias de la Universidad de Las Américas, sede Viña del Mar (Chile), entre enero de 2018 y septiembre de 2019. El protocolo de trabajo con animales fue aprobado por el Comité de Ética Científica de la Universidad de las Américas.

La información recolectada incluyó peso vivo (PV) y edad (E). El PV se consideró relevante en función de la heterogeneidad de tallas que se pueden observar en perros mestizos, y de acuerdo con el PV y E de los ejemplares, se conformaron seis grupos:

- PVIEl: perros pequeños, menores de $15 \mathrm{~kg}$ y $>1$ y $\leq 5$ años $(\mathrm{n}=15)$

- PV1E2: perros pequeños, menores de $15 \mathrm{~kg}$ y $>5$ años $(\mathrm{n}=15)$.

- PV2E1: perros medianos, entre 15 y $25 \mathrm{~kg}$ y $>1$ y $\leq 5$ años $(\mathrm{n}=15)$.

- PV2E2: perros medianos, entre 15 y $25 \mathrm{~kg}$ y $>5$ años $(n=15)$.

- PV3El: perros grandes, mayores de $25 \mathrm{~kg}$ y $>1$ y $\leq 5$ años $(n=15)$.

- PV3E2: perros grandes, mayores de $25 \mathrm{~kg}$ y $>5$ años $(n=15)$.

El examen físico reproductivo incluyó palpación e inspección de pene y prepucio, inspección y medición de perímetro escrotal, palpación testicular y palpación de epidídimos.

Los exámenes ultrasonográficos se realizaron por el mismo operador con el paciente en estación, sin necesidad de sedación, utilizando un equipo Mindray M5Vet con transductor microconvexo de 5 a $8 \mathrm{MHz}$.

La evaluación testicular se efectuó en los planos longitudinal y transversal, y consideró ecogenicidad del parénquima, visualización de mediastino y presencia o ausencia de estructuras anecoicas y/o hiperecoicas.

La evaluación prostática se realizó en los planos longitudinal y transversal, y consideró situación, tamaño, forma, simetría, estructura capsular y ecotextura del parénquima, contorno, presencia y cantidad de focos anecoicos e hiperecoicos.

Además se evaluó el diámetro glandular ${ }^{1}$ en el plano transversal (Rev Inv Vet Perú 30: 1249-1256). Con las variables continuas se realizó estadística descriptiva, y para variables categóricas se estimaron las frecuencias.

Para variables continuas se utilizó el test de Student y para variables categóricas se empleó el test no paramétrico Mann-Whitney. Se ocupó el programa STATA 14 , considerando un $95 \%$ de confianza $(p<0,05)$.

\section{RESULTADOS Y DISCUSIÓN}

Las características de peso vivo y edad de los ejemplares, para los distintos grupos, se presentan en la Tabla 1, destacando que entre los grupos de distinto peso vivo se observaron diferencias, independientes de la edad y entre los grupos de diferente edad se observaron diferencias, independientes del peso vivo $(p<0,05)$.

Cabe considerar que en el examen físico, se considera que los testículos normales deben ser simétricos en tamaño y forma, de consistencia elástica y capacidad de desplazarse libremente en el escroto ${ }^{15}$. 
Tabla 1. Promedio \pm desviación estándar de peso vivo y edad en perros mestizos enteros sometidos a examen de salud reproductiva.

\begin{tabular}{lccc}
\hline grupo & $\mathrm{n}$ & peso vivo $(\mathrm{kg})$ & edad (años) \\
\hline PV1E1 & 15 & $8,96 \pm 1,84^{\mathrm{a}}$ & $2,86 \pm 0,76^{\mathrm{a}}$ \\
PV1E2 & 15 & $9,60 \pm 2,10^{\mathrm{a}}$ & $8,16 \pm 1,52^{\mathrm{b}}$ \\
PV2E1 & 15 & $18,62 \pm 2,46^{\mathrm{b}}$ & $2,96 \pm 0,87^{\mathrm{a}}$ \\
PV2E2 & 15 & $18,14 \pm 1,52^{\mathrm{b}}$ & $8,53 \pm 1,79^{\mathrm{b}}$ \\
PV3E1 & 15 & $33,23 \pm 5,84^{\mathrm{c}}$ & $3,20 \pm 1,13^{\mathrm{a}}$ \\
PV3E2 & 15 & $31,72 \pm 3,83^{\mathrm{c}}$ & $8,10 \pm 1,21^{\mathrm{b}}$ \\
\hline
\end{tabular}

a,b Superíndices distintos dentro de columnas indican diferencias significativas $(\mathrm{p}<0,05)$

En el presente examen de salud reproductiva se registraron mayores frecuencias de anomalías testiculares, entre ellas asimetría y tono disminuido (flaccidez). en perros $>5$ años, independientes del peso vivo, respecto de animales más jóvenes $(\mathrm{p}<0,05)$.

Lo anterior se puede relacionar con una disminución del parénquima gonadal, fenómeno asociado a degeneración y atrofia testicular ${ }^{11}$. Por otra parte, el perímetro escrotal no arrojó diferencias al comparar perros de los mismos grupos de peso vivo, independientes de la edad, con valores considerados como normales para el peso de los ejemplares ${ }^{19}$.

En la evaluación ultrasonográfica testicular, se observó mayor frecuencia de alteraciones de parénquima, especialmente heterogeneidad y presencia de focos anecoicos en perros $>5$ años, independientes del peso vivo $(\mathrm{p}<0,05)$, lo cual guardaría relación con que los cambios degenerativos en los testículos caninos son más pronunciados sobre los 8 años de edad ${ }^{3}$.

Además, se ha descrito que los cambios macroscópicos no serían evidentes en los grados leves a moderados de degeneración, a diferencia de lo que ocurre en los perros de mayor edad, donde se observa una disminución del tamaño testicular debido a una degeneración avanzada ${ }^{11}$.
Dado que los grupos de perros $>5$ años presentaron promedios de edad superiores a 8 años, se los podría considerar como animales viejos ${ }^{13}$, resultando concordantes las observaciones del examen físico reproductivo con el examen ultrasonográfico testicular.

Cabe destacar que los ejemplares examinados en nuestro estudio (criterio de inclusión), se presentaron sin signos evidentes de compromiso prostático tales como hematuria, disuria, constipación o tenesmo ${ }^{9}$.

Sin embargo, en la evaluación ecográfica de próstata (Tabla 2), destacó la alta proporción de anomalías de ecotextura, asimetría lobular, contorno glandular irregular y presencia de focos anecoicos en los grupos de perros $>5$ años $(\mathrm{p}<0,05)$, descripción que coincide con otros autores ${ }^{5}$.

En la Tabla 3 se presentan el promedio y la desviación estándar del diámetro prostático transverso, en perros mestizos de acuerdo con el peso vivo y la edad, para los diferentes grupos en estudio. En el análisis de esta variable, se observaron mayores valores en todos los grupos de perros $>5$ años, independiente del peso vivo $(p<0,05)$.

Cabe destacar que la prostatomegalia se observó en el $88,8 \%$ de perros $>5$ años, valor significativamente superior $(p<0,05)$ a lo observado en ejemplares más jóvenes $(20 \%)$, independientes del peso vivo. Esto es similar a lo descrito en un estudio retrospectivo, donde se destaca que el promedio de edad en que los perros experimentan de-sórdenes prostáticos es de $8,6 \pm 3,2$ años, siendo $6,2 \pm 2,3$ la edad en que se diagnostica más comúnmente la hiperplasia prostática benigna (HPB) en pacientes asintomáticos ${ }^{14}$.

Relacionando la compatibilidad de las alteraciones ultrasonográficas con tipos de patologías específicas de la próstata, podríamos inferir la presencia de HPB, la cual coincidió en cerca de un $70 \%$ de los casos con presencia de estructuras quísticas únicas o múltiples en el parénquima glandular ${ }^{8,14}$.

Tabla 2. Frecuencias de alteraciones prostáticas, registradas mediante ultrasonografía en perros mestizos enteros según grupo de estudio.

\begin{tabular}{lcccccc}
\hline \multirow{2}{*}{ variable prostática } & \multicolumn{3}{c}{ grupo de estudio (peso vivo/edad) } \\
\cline { 2 - 7 } & PV1E1 & PV1E2 & PV2E1 & PV2E2 & PV3E1 & PV3E2 \\
\hline anomalías de ecotextura & $0,13^{\mathrm{a}}$ & $0,80^{\mathrm{b}}$ & $0,20^{\mathrm{a}}$ & $0,66^{\mathrm{b}}$ & $0,20^{\mathrm{a}}$ & $0,80^{\mathrm{b}}$ \\
asimetría lobular & $0,13^{\mathrm{a}}$ & $0,80^{\mathrm{b}}$ & $0,06^{\mathrm{a}}$ & $0,93^{\mathrm{b}}$ & $0,13^{\mathrm{a}}$ & $0,80^{\mathrm{b}}$ \\
contorno irregular & $0,13^{\mathrm{a}}$ & $0,86^{\mathrm{b}}$ & $0,06^{\mathrm{a}}$ & $0,86^{\mathrm{b}}$ & $0.06^{\mathrm{a}}$ & $0,66^{\mathrm{b}}$ \\
focos anecoicos & $0,06^{\mathrm{a}}$ & $0,66^{\mathrm{b}}$ & $0,13^{\mathrm{a}}$ & $0,53^{\mathrm{b}}$ & $0,13^{\mathrm{a}}$ & $0,66^{\mathrm{b}}$ \\
aumento de tamaño & $0,20^{\mathrm{a}}$ & $0,93^{\mathrm{b}}$ & $0,20^{\mathrm{a}}$ & $0,80^{\mathrm{b}}$ & $0,20^{\mathrm{a}}$ & $0,93^{\mathrm{b}}$ \\
\hline
\end{tabular}

a, b: superíndices distintos dentro de filas indican diferencias significativas $(p<0,05)$.

Tabla 3. Promedio \pm desviación estándar de diámetro prostático en perros mestizos de acuerdo con el peso vivo y la edad.

\begin{tabular}{|c|c|c|c|c|c|c|}
\hline \multirow{2}{*}{ variable prostática } & \multicolumn{6}{|c|}{ grupo de estudio (peso vivo/edad) } \\
\hline & PV1E1 & PV1E2 & PV2E1 & PV2E2 & PV3E1 & PV3E2 \\
\hline diámetro transverso $(\mathrm{cm})$ & $2,76 \pm 1,15$ & $3,72 \pm 0,23$ & $3,29 \pm 0,65$ & $4,38 \pm 0,73$ & $4,16 \pm 0,55$ & $5,28 \pm 0,98$ \\
\hline
\end{tabular}


Dicha condición se describe habitualmente en perros enteros mayores a los 5 años de edad ${ }^{2,16}$, con una prevalencia de $90 \%$ para individuos de entre 7,1 y 8 años ${ }^{4}$. Dado lo anterior, resulta interesante destacar que la incidencia de enfermedades prostáticas ha aumentado en los últimos años debido al incremento en las expectativas de vida de los perros, estimándose un promedio de 11 a 12 años de existencia ${ }^{10,13}$.

En concordancia con la hipótesis propuesta para el presente estudio, los perros mestizos enteros $>5$ años, presentaron mayores proporciones de alteraciones testiculares y prostáticas subclínicas respecto de animales más jóvenes, independientemente de su peso vivo.

Tales alteraciones podrían derivar en detrimento del bienestar de los animales, postulando con ello la importancia de establecer pautas en salud reproductiva de perros mestizos enteros, a fin de mejorar la calidad de vida de los mismos. Por ejemplo, educando a los propietarios en la conveniencia de la castración temprana de ejemplares sin propósitos de cría, teniendo en cuenta que la medicina veterinaria organizada puede contribuir sustancialmente en la educación de la comunidad ${ }^{17}$.

Ello asume importancia considerando especialmente el elevado porcentaje de perros mestizos enteros en la ciudad de Viña del Mar ${ }^{12}$, que exige generar recomendaciones a los médicos veterinarios para la práctica de estudios preventivos.

Por otra parte, con el fin de detectar tempranamente anormalidades prostáticas, se ha propuesto que los exámenes preventivos deberían comenzar a realizarse luego que el perro cumpla el $40 \%$ de su expectativa de vida ${ }^{10}$. Por lo tanto, se considera que el concepto "salud reproductiva" debiera ser parte de la medicina veterinaria preventiva.

De los resultados obtenidos en el presente estudio se puede concluir que perros mestizos enteros $>5$ años, independientemente de su peso vivo, presentaron mayores proporciones de alteraciones testiculares y prostáticas subclínicas, respecto de animales más jóvenes, denotando una afección de su salud reproductiva. Ello plantea el desafío de concentrar las actividades hacia las medidas preventivas, esperando que las afecciones disminuyan a favor del bienestar de los caninos.

Agradecimientos. A la Dirección de Investigación de la Universidad de Las Américas por el financiamiento a través del Proyecto Interno 2018028.

\section{REFERENCIAS}

1. Atalan G, Holt P, Barr J, Brown P. 1999. Ultrasonographic estimation of prostatic size in canine cadavers. Res Vet Sci 67: 7-15.

2. Barsanti J, Finco D. 2002. Enfermedades prostáticas caninas. En: Ettinger S. \& Feldman E. (editores): Tratado de medicina interna veterinaria, $6^{\circ}$ ed., Elsevier, España, p. 1952-1971.
3. Bhanmeechao C, Srisuwatanasagul S, Ponglowhapan S. 2018. Age-related changes in interstitial fibrosis and germ cell degeneration of the canine testis. Reprod Domest Anim 53: 37-43

4. Christensen B. 2018. Canine prostate disease. Vet Clin Small Anim 48: 701-719.

5. Dawkins M. 2007. Through animal eyes: what behaviour tells us. Appl Anim Behav Sci 100: 4-10.

6. Grieco V et al. 2008. Canine testicular tumours: a study on 232 dogs. J Comp Path 138: 86-89.

7. Ingus C. 2018. Guia de perro mestizo chileno, Edit. Planeta, Chile, p. 71-82.

8. Johnston S, Rootkrustritz M, Olson P. 2001. Canine and feline theriogenology (Disorders of the canine prostate), Ed. Saunders, Philadelphia, p. 337-355.

9. Lévy X, Nizanski W, Heimendahl A, Mimouni P. 2014. Diagnosis of common prostatic conditions in dogs: an update. Reprod Domest Anim 49: 50-57.

10. Mantziaras G, Alonge S, Faustini M, Luvoni G. 2017. Assessment of age for a preventive ultrasonographic examination of the prostate in the dog. Theriogenology 100 : 114-119.

11. McEntee K. 1990. Reproductive pathology of domestic mammals, Academic Press, San Diego, California (USA), $401 \mathrm{p}$.

12. Morales M, Varas C, Ibarra L. 2009. Caracterización demográfica de la población de perros de Viña del Mar, Chile. Arch Med Vet 41: 89-95.

13. O’Neill D, Church D, McGreevy P, Thompson P, Brodbelt D. 2013. Longevity and mortality of owned dogs in England. Vet $J$ 198: 638-643.

14. Polisca A, Troisi A, Fontain E, Menchetti L, Fontbonne A. 2016. A retrospective study of canine prostatic disease from 2002 to 2009 at the Alfort Veterinary College in France. Theriogenology 85: 835-840.

15. Root KM. 2014. Applied small animal andrology. In: Animal andrology: theories and applications (Chenoweth $\mathrm{P}$, Lorton S), Cab International, Wallingford, England, p. 177-196.

16. Ruel Y, Barthez P, Mailles A, Begon D. 1998. Ultrasonographic evaluation of the prostate in healthy intact dogs. Vet Radiol Ultrasoun 39: 212-216.

17. Salamanca C, Polo L, Vargas J. 2011. Sobrepoblación canina y felina: tendencias y nuevas perspectivas. Rev Med Vet Zoot 58: 45-53.

18. Smith J. 2008. Canine prostatic disease: a review of anatomy, pathology, diagnosis, and treatment. Theriogenology 70: 375-383.

19. Woodall P, Johnstone I. 1988. Dimentions and allometry of testes, epididymides and spermatozoa in the domestic dog. J Reprod Fertil 82: 603-609.

20. Ygreda G, Grandez R, Valencia R. 2017. Caracterización de las alteraciones ultrasonográficas prostáticas en perros atendidos en una clínica veterinaria en Lima, Perú, entre 2010 y 2014. Rev Inv Vet Perú 28: 299-306.

21. Yeates JW. 2012. Maximizing canine welfare in veterinary practice and research: a review. The Vet $J$ 192: 272278. 